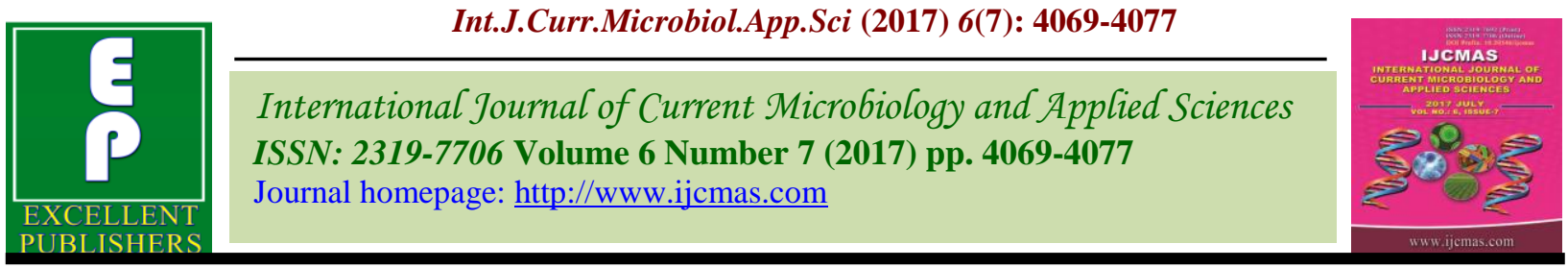

Original Research Article

https://doi.org/10.20546/ijcmas.2017.607.422

\title{
Studies on Heterosis in Bitter Gourd (Momordica charantia L.)
}

\author{
L. Bhatt ${ }^{1 *}$, S.P. Singh ${ }^{1}$, A.K. Soni ${ }^{1}$ and M.K. Samota ${ }^{2}$ \\ ${ }^{1}$ Sri Karan Narendra Agriculture University, Jobner-303329, India \\ ${ }^{2}$ ICAR- IARI, New Delhi-110 012, India \\ *Corresponding author
}

\begin{tabular}{|c|c|}
\hline & A B S T R A C T \\
\hline & $\begin{array}{l}\text { The present investigation entitled studies on heterosis for yield and its components of bitter } \\
\text { gourd (Momordica charantia L.) using } 9 \text { parents and } 1 \text { standard variety and their } 36 \\
\text { crosses for } 11 \text { quantitive traits was carried out in summer and rainy seasons of } 2015 \text {. The } \\
\text { analysis of variance for experimental design revealed the existence of adequate genetic } \\
\text { variability in experimental material for all traits under study. Variance due to parents Vs }\end{array}$ \\
\hline Keywords & F1's were significant for all characters, except days taken to opening of first female \\
\hline $\begin{array}{l}\text { Bitter gourd, } \\
\text { Momordica } \\
\text { charantia, } \\
\text { Heterosis and } \\
\text { Harvest }\end{array}$ & $\begin{array}{l}\text { flower,days taken to first fruit set, fruit weight and total fruits yield per vine, thereby } \\
\text { indicating the presence of overall average heterosis for all characters. Heterosis was } \\
\text { worked -out over better parent and standard check Pusa hybrid-1. The highest desirable } \\
\text { heterobeltiosis for yield its component characters was observed in order of male: female } \\
\text { ratio }(37.36 \% \text { ), number fruit per vine }(20.00 \%) \text {, total fruits yield per vine }(9.68 \%) \text { and }\end{array}$ \\
\hline Article Info & in order of days taken to opening of first female flower(-5.45), days taken to first fruits set \\
\hline $\begin{array}{l}\text { Accepted: } \\
\text { 29 June } 2017 \\
\text { Available Online: } \\
\text { 10 July } 2017\end{array}$ & $\begin{array}{l}\text { (-5.45), male: female ratio }(107.47 \%) \text {, number of fruits per vine }(50.00 \%) \text {, fruit length } \\
(27.59 \%) \text {, fruit weight }(3.33) \text {, fruit diameter }(4.35 \%) \text {, total fruits yield per vine }(21.43 \%) \text {. } \\
\text { Panipat Local x Phule Green, Phule Green x Pusa Do Mausami and Punjab-14 x Pusa Do } \\
\text { Mausami. Panipat Local x Phule Green was superior in respect of days taken to opening } \\
\text { first female flower, vine length, days to first fruit harvest, fruit length. Phule Green x Pusa }\end{array}$ \\
\hline & $\begin{array}{l}\text { Do Mausami was took fruit length, fruit weight, fruit diameter and total fruits yield per } \\
\text { vine. Punjab-14 x Pusa Do Mausami was better with regard to superior for number of node } \\
\text { at which first female flower appeared, number of fruits per vine and days to first fruit } \\
\text { harvest. }\end{array}$ \\
\hline
\end{tabular}

\section{Introduction}

Bitter gourd (Momordica charantia L.) is an important nutritive and commercial cucurbits belonging to the Cucurbitaceae. In terms of nutritive value, bitter gourd ranks first among cucurbits due to high value of vitamins and minerals. It is highly cross pollinated due to monoecious nature and has high degree of heterozygosity. Due to efforts of vegetable breeders, improved varieties and hybrids have been developed. Crops improvement involves strategies for enhancing yield potentiality and quality components. In bitter gourd full exploitation of heterosis through development of hybrids has not been successfully commercialized due to one or the other reason. Variability found in shape, size and colour of fruits in most conspicuous which offers tremendous scope for heterosis breeding for yield enhancement. Crossing nature and heterosis in cross pollinated crops 
has been known to offer good potentialities for increased yield.

\section{Materials and Methods}

The present investigation was undertaken involving different diverse genotypes/cultivars of bitter gourd were crossed in all possible combinations excluding reciprocals. The $36 \mathrm{~F}_{1}$ hybrids along with their nine parents were evaluated during kharif 2015 in a RBD with three replications at Horticulture Farm, S.K.N. College of Agriculture, Jobner Rajasthan. Observation were recorded on five randomly selected tagged plants from each treatment for yield and yield attributing traits viz., vine Length $(m)$, days taken to opening of first female flower, days taken to first fruits set, number of node at which first female flower appeared, male : female ratio, number of fruit per vine, days to first fruit harvest, fruit length (cm) at harvest, fruit weight $(\mathrm{g})$, fruit diameter $(\mathrm{cm})$, total fruits yield per vine $(\mathrm{kg})$ and ascorbic acid (mg)/100 (g). Heterosis over better parents and commercial check, were computed following standard statistical procedure Fonseca and Patterson (1968) was applied to compute heterosis estimates.

\section{Results and Discussion}

The analysis of variance for experimental design revealed the existence of adequate genetic variability in experimental material for all traits under study. Variance due to parents Vs F1's were significant for all characters, except days taken to opening of first female flower, days taken to first fruit set, fruit weight and total fruits yield per vine, thereby indicating the presence of overall average heterosis for all characters (Table 1). Heterosis was computed as per cent increase or decrease in $\mathrm{F}_{1}$ valves over the better parents used over the best standard (SV) (Table 2). In the present investigation the relative magnitude of heterosis over the better parents and standard variety (Pusa Hybrid-1) was studied for 11 characters (Table 1) days taken to openings of first female flower revealed that high degree of standard heterosis for in cross combination Punjab-14 x Pant karela-1 and kalyanpur Sona $\mathrm{x}$ Pant karela-1.The heterosis over better parent and standard variety for days taken to first fruit set among the cross revealed that 9 cross showed negative significant heterosis over the better parents, high degree of standard heterosis was observed in Punjab-14 x Pant karela-1 and kalyanpur Sona x Pant karela-1. These results are in conformity with those of Verma and Singh (2014).For number of node at which first female flower appeared heterosis over better parent and all 36 crosses and standard variety showed highly significant heterosis in negative direction by Ram et al., (1999).

For vine length 17 crosses showed over better parent and 27 crosses standard variety highly significant heterosis in negative direction. The cross Punjab-14 x kalyanpur Baramasi showed highly significant heterosis both over better parent and standard variety. Conformity evidence for above mentioned characters has also been given by Singh et al., (2013) and Kumara et al., (2011). For male: Female 10 cross over better parent and 21 crosses standard variety highly significant heterosis in positive direction. The Highest heterosis was observed over better parent in cross Phule Green x Pant Karela-1 and Arka Harit x Phule Green over standard variety (Thangamani et al., 2011). For number of fruit per plant heterosis was showed by 5 cross over better parent and 19 cross over standard variety. The Highest standard heterosis Punjab-14 x Pusa Vishesh, Punjab-14 x Pusa Do Mousami, kalyanpur Baramasi x kalyanpur Sona, kalyanpur Sona x Pant Karela-1 for number of fruits per plant was recorded (Mangal et al., 1984 and Khattra et al., 2000). 
Table.1 Analysis of variance showing mean sum of square for different characters in bitter gourd

\begin{tabular}{|c|c|c|c|c|c|c|c|c|}
\hline \multirow[t]{3}{*}{ S.No. } & \multirow[t]{3}{*}{ Characters } & \multirow{3}{*}{ d.f } & \multicolumn{6}{|c|}{ Source } \\
\hline & & & Replication & Genotypes & Parents & $\mathbf{F}_{1} \mathbf{S}$ & P Vs $\mathbf{F}_{1}$ & Error \\
\hline & & & 2 & 44 & 8 & 35 & 1 & 88 \\
\hline 1 & $\begin{array}{l}\text { Days taken to opening of first female } \\
\text { Flower }\end{array}$ & & 10.722 & $41.066^{* *}$ & $51.083 * *$ & $39.333 * *$ & 21.604 & 6.683 \\
\hline 2 & Days taken to first fruit set & & 22.176 & $35.948 * *$ & $49.833 * *$ & $33.454 * *$ & 12.153 & 7.233 \\
\hline 3 & $\begin{array}{l}\text { Number of node at which first female } \\
\text { flower appeared }\end{array}$ & & 0.286 & $1.521 * *$ & $0.750 * *$ & $1.569 * *$ & $6.016^{* *}$ & 0.130 \\
\hline 4 & Vine length (m) at harvest & & 0.043 & $0.163 * *$ & $0.124 * *$ & $0.159 * *$ & $0.598 * *$ & 0.036 \\
\hline 5 & Male:female ratio & & 0.448 & $46.933 * *$ & $25.418 * *$ & $48.623 * *$ & $159.870 * *$ & 3.572 \\
\hline 6 & Number of fruit/ vine & & 1.274 & $13.492 * *$ & $8.250 * *$ & $14.423 * *$ & $22.820 * *$ & 1.577 \\
\hline 7 & Days to first fruit harvest & & 20.545 & $128.918 * *$ & $198.750 * *$ & $115.542 * *$ & $38.399 *$ & 7.955 \\
\hline 8 & Fruit length $(\mathrm{cm})$ at harvest & & 2.814 & $35.456^{* *}$ & $78.870 * *$ & $25.800 * *$ & $26.136 * *$ & 1.845 \\
\hline 9 & Fruit weight (g) & & 13.926 & $52.145^{* *}$ & $98.333 * *$ & $42.590 * *$ & 17.066 & 8.490 \\
\hline 10 & Fruit diameter (cm) & & 0.069 & $0.817 * *$ & $0.930 * *$ & $0.702 * *$ & $3.950 * *$ & 0.144 \\
\hline 11 & Total fruits yield per vine(kg) & & 0.002 & $0.020 * *$ & $0.031 * *$ & $0.017 * *$ & 0.006 & 0.002 \\
\hline 12 & Ascorbic acid (mg/100 g) & & 0.003 & $0.015^{* *}$ & $0.022 * *$ & $0.012 * *$ & $0.091 * *$ & 0.003 \\
\hline
\end{tabular}


Table.2 Magnitude of heterosis (\%) over better parent and commercial check for various yield and yield parameters in bitter gourd

\begin{tabular}{|c|c|c|c|c|c|c|c|c|}
\hline \multirow[t]{2}{*}{ Hybrids } & \multicolumn{2}{|c|}{$\begin{array}{l}\text { Days taken to opening of first } \\
\text { female flower }\end{array}$} & \multicolumn{2}{|c|}{$\begin{array}{c}\text { Days taken to first } \\
\text { fruit set }\end{array}$} & \multicolumn{2}{|c|}{$\begin{array}{c}\text { Number of node at which first } \\
\text { female flower appeared }\end{array}$} & \multicolumn{2}{|c|}{$\begin{array}{l}\text { Vine length at } \\
\text { harvest }(\mathrm{m})\end{array}$} \\
\hline & $\mathrm{BP}$ & $\mathrm{CC}$ & $\mathrm{BP}$ & $\mathrm{CC}$ & $\mathrm{BP}$ & $\mathrm{CC}$ & $\mathrm{BP}$ & $\mathrm{CC}$ \\
\hline Punjab-14 x Arka Harit & $-11.90 *$ & $-32.73 * *$ & -9.30 & $-29.09 * *$ & $-20.00 * *$ & $-42.86 * *$ & 0.00 & 0.00 \\
\hline Punjab-14 x Panipat Local & $-10.64 *$ & $-23.64 * *$ & $-10.64 *$ & $-23.64 * *$ & $-20.00 * *$ & $-42.86 * *$ & $-12.50 *$ & $-12.50 *$ \\
\hline Punjab-14 x Phule Green & -8.00 & $-16.36 * *$ & -4.00 & $-12.73 * *$ & $20.00 * *$ & $-14.29 * *$ & -6.25 & -6.25 \\
\hline Punjab-14 x Pusa Vishesh & $-10.00 *$ & $-18.18 * *$ & -8.00 & $-16.36 * *$ & $-20.00 * *$ & $-42.86 * *$ & $-12.50 *$ & $-12.50 *$ \\
\hline $\begin{array}{l}\text { Punjab-14 x Pusa Do } \\
\text { Mausami }\end{array}$ & -8.00 & $-16.36^{* *}$ & $-9.80 *$ & $-16.36^{* *}$ & 0.00 & $-28.57 * *$ & $-18.75^{* *}$ & $-18.75^{* *}$ \\
\hline $\begin{array}{l}\text { Punjab-14 x Kalyanpur } \\
\text { Barahmasi }\end{array}$ & -2.22 & $-20.00 * *$ & 0.00 & $-18.18 * *$ & $-40.00 * *$ & $-57.14 * *$ & $-31.25 * *$ & $-31.25 * *$ \\
\hline $\begin{array}{l}\text { Punjab-14 x Kalyanpur } \\
\text { Sona }\end{array}$ & 0.00 & $-27.27 * *$ & 2.44 & $-23.64 * *$ & $-20.00 * *$ & $-42.86 * *$ & $-18.75 * *$ & $-18.75^{* *}$ \\
\hline Punjab-14 x Pant Karela-1 & $-21.74 * *$ & $-34.55 * *$ & $-22.92 * *$ & $-32.73 * *$ & $-20.00 * *$ & $-42.86 * *$ & $-12.50 *$ & $-12.50 *$ \\
\hline $\begin{array}{l}\text { Arka Harit x Panipat } \\
\text { Local }\end{array}$ & $-10.64 *$ & $-23.64 * *$ & -8.51 & $-21.82 * *$ & $-20.00 * *$ & $-42.86 * *$ & $-13.33^{*}$ & -18.75 \\
\hline Arka Harit x Phule Green & $-10.01 *$ & $-18.19 * *$ & $-10.01 *$ & $-18.19 * *$ & $-20.00 * *$ & $-42.86 * *$ & -6.67 & $-12.50 *$ \\
\hline Arka Harit x Pusa Vishesh & $-10.00 *$ & $-18.18 * *$ & -8.00 & $-16.36^{* *}$ & $-25.00 * *$ & $-57.14 * *$ & $-20.00 * *$ & $-25.00 * *$ \\
\hline $\begin{array}{l}\text { Arka Harit x Pusa Do } \\
\text { Mausami }\end{array}$ & -8.00 & $-16.36^{* *}$ & $-9.80 *$ & $-16.36 * *$ & $-20.00 * *$ & $-42.86 * *$ & -7.14 & $-18.75 * *$ \\
\hline $\begin{array}{l}\text { Arka Harit x Kalyanpur } \\
\text { Barahmasi }\end{array}$ & -6.67 & $-23.64 * *$ & -2.22 & $-20.00 * *$ & $-20.00 * *$ & $-42.86 * *$ & $-13.33^{*}$ & $-18.75^{* *}$ \\
\hline $\begin{array}{l}\text { Arka Harit x Kalyanpur } \\
\text { Sona }\end{array}$ & 0.00 & $-23.64 * *$ & 2.33 & $-20.00 * *$ & $25.00 * *$ & $-28.57 * *$ & 7.69 & $-12.50 *$ \\
\hline $\begin{array}{l}\text { Arka Harit x Pant Karela- } \\
1\end{array}$ & $-10.87 *$ & $-25.45 * *$ & $-12.50 * *$ & $-23.64 * *$ & $-40.00 * *$ & $-57.14 * *$ & $-14.29 *$ & $-25.00 * *$ \\
\hline $\begin{array}{l}\text { Panipat Local x Phule } \\
\text { Green }\end{array}$ & -4.00 & $-12.73 * *$ & -4.00 & $-12.73 * *$ & 0.00 & $-28.57 * *$ & 0.00 & -6.25 \\
\hline $\begin{array}{l}\text { Panipat Local x Pusa } \\
\text { Vishesh }\end{array}$ & $-12.00 * *$ & $-20.00 * *$ & $-10.00^{*}$ & $-18.18 * *$ & $-20.00 * *$ & $-42.86 * *$ & -6.67 & $-12.50 *$ \\
\hline $\begin{array}{l}\text { Panipat Local x Pusa Do } \\
\text { Mausami }\end{array}$ & -8.00 & $-16.36^{* *}$ & $-9.80 *$ & $-16.36^{* *}$ & 0.00 & $-28.57 * *$ & 0.00 & -6.25 \\
\hline Panipat Local $\mathrm{x}$ & -4.26 & $-18.18 * *$ & -4.26 & $-18.18 * *$ & $-20.00 * *$ & $-42.86 * *$ & -6.67 & $-12.50^{*}$ \\
\hline
\end{tabular}


Int.J.Curr.Microbiol.App.Sci (2017) 6(7): 4069-4077

\begin{tabular}{|c|c|c|c|c|c|c|c|c|}
\hline Kalyanpur Barahmasi & & & & & & & & \\
\hline $\begin{array}{l}\text { Panipat Local } \mathrm{x} \\
\text { Kalyanpur Sona }\end{array}$ & -6.38 & $-20.00 * *$ & -4.26 & $-18.18 * *$ & $-20.00 * *$ & $-42.86^{* *}$ & $-13.33 *$ & $-18.75^{* *}$ \\
\hline $\begin{array}{l}\text { Panipat Local x Pant } \\
\text { Karela-1 }\end{array}$ & -6.38 & $-20.00 * *$ & -6.25 & $-18.18 * *$ & $-20.00 * *$ & $-42.86 * *$ & -6.67 & -12.50 \\
\hline $\begin{array}{l}\text { Phule Green x Pusa } \\
\text { Vishesh }\end{array}$ & 0.00 & $-9.09 *$ & 0.00 & $-9.09 *$ & 0.00 & $-28.57 * *$ & 6.67 & 0.00 \\
\hline $\begin{array}{l}\text { Phule Green x Pusa Do } \\
\text { Mausami }\end{array}$ & -4.00 & $-12.73 * *$ & -1.96 & $-9.09 *$ & $-20.00 * *$ & $-42.86 * *$ & -6.67 & $-12.50 *$ \\
\hline $\begin{array}{l}\text { Phule Green x Kalyanpur } \\
\text { Barahmasi }\end{array}$ & 0.00 & $-9.09 *$ & 0.00 & $-9.09 *$ & $-20.00 * *$ & $-42.86 * *$ & -6.67 & $-12.50 *$ \\
\hline $\begin{array}{l}\text { Phule Green x Kalyanpur } \\
\text { Sona }\end{array}$ & -6.00 & $-14.55^{* *}$ & -4.00 & $-12.73 * *$ & $-20.00 * *$ & $-42.86 * *$ & $-13.33 *$ & $-18.75 * *$ \\
\hline $\begin{array}{l}\text { Phule Green x Pant } \\
\text { Karela-1 }\end{array}$ & $-10.00^{*}$ & $-18.18 * *$ & -8.00 & $-16.36^{* *}$ & $-40.00 * *$ & $-57.14 * *$ & $-20.00 * *$ & $-25.00 * *$ \\
\hline $\begin{array}{l}\text { Pusa Vishesh x Pusa Do } \\
\text { Mausami }\end{array}$ & 4.00 & -5.45 & 1.96 & -5.45 & 0.00 & $-28.57 * *$ & 0.00 & -6.25 \\
\hline $\begin{array}{l}\text { Pusa Vishesh x Kalyanpur } \\
\text { Barahmasi }\end{array}$ & -4.00 & $-12.73 * *$ & -4.00 & $-12.73 * *$ & $-20.00 * *$ & $-42.86 * *$ & -6.67 & $-12.50 *$ \\
\hline $\begin{array}{l}\text { Pusa Vishesh x Kalyanpur } \\
\text { Sona }\end{array}$ & -6.00 & $-14.55 * *$ & -4.00 & $-12.73 * *$ & $25.00 * *$ & $-28.57 * *$ & -6.67 & $-12.50^{*}$ \\
\hline $\begin{array}{l}\text { Pusa Vishesh x Pant } \\
\text { Karela-1 }\end{array}$ & -8.00 & $-16.36 * *$ & -6.00 & $-14.55 * *$ & $-20.00 * *$ & $-42.86 * *$ & $-13.33 *$ & $-18.75^{* *}$ \\
\hline $\begin{array}{l}\text { Pusa Do Mausami x } \\
\text { Kalyanpur Barahmasi }\end{array}$ & -4.00 & $-12.73 * *$ & -5.88 & $-12.73 * *$ & 0.00 & $-28.57 * *$ & 0.00 & -6.25 \\
\hline $\begin{array}{l}\text { Pusa Do Mausami x } \\
\text { Kalyanpur Sona }\end{array}$ & -8.00 & $-16.36^{* *}$ & -7.84 & $-14.55^{* *}$ & $-20.00 * *$ & $-42.86^{* *}$ & -7.14 & $-18.75^{* *}$ \\
\hline $\begin{array}{l}\text { Pusa Do Mausami x Pant } \\
\text { Karela-1 }\end{array}$ & $-10.00 *$ & $-18.18 * *$ & $-9.80^{*}$ & $-16.36 * *$ & $-40.00 * *$ & $-57.14 * *$ & $-14.29 *$ & $-25.00 * *$ \\
\hline $\begin{array}{l}\text { Kalyanpur Barahmasi x } \\
\text { Kalyanpur Sona }\end{array}$ & -6.67 & $-23.64 * *$ & -4.44 & $-21.82 * *$ & $-20.00 * *$ & $-42.86 * *$ & $-13.33 *$ & $-18.75^{* *}$ \\
\hline $\begin{array}{l}\text { Kalyanpur Barahmasi x } \\
\text { Pant Karela-1 }\end{array}$ & -4.35 & $-20.00 * *$ & -8.33 & $-20.00 * *$ & 0.00 & $-28.57 * *$ & -6.78 & $-12.60 *$ \\
\hline $\begin{array}{l}\text { Kalyanpur Sona x Pant } \\
\text { Karela-1 }\end{array}$ & $-21.74 * *$ & $-34.55^{* *}$ & $-22.92 * *$ & $-32.73 * *$ & $-40.00 * *$ & $-57.14 * *$ & $-14.29 *$ & $-25.00 * *$ \\
\hline
\end{tabular}




\begin{tabular}{|c|c|c|c|c|c|c|c|c|}
\hline \multirow[t]{2}{*}{ Hybrids } & \multicolumn{2}{|c|}{ Male : Female ratio } & \multicolumn{2}{|c|}{$\begin{array}{c}\text { Number of fruits per } \\
\text { vine }\end{array}$} & \multicolumn{2}{|c|}{$\begin{array}{c}\text { Days to first fruit } \\
\text { harvest }\end{array}$} & \multicolumn{2}{|c|}{ Fruit length at harvest $(\mathrm{cm})$} \\
\hline & $\mathrm{BP}$ & $\mathrm{CC}$ & $\mathrm{BP}$ & $\mathrm{CC}$ & $\mathrm{BP}$ & $\mathrm{CC}$ & $\mathrm{BP}$ & $\mathrm{CC}$ \\
\hline Punjab-14 x Arka Harit & $28.57 * *$ & 7.88 & 13.33 & $41.67 * *$ & -3.45 & 7.69 & -9.86 & $-26.44 * *$ \\
\hline Punjab-14 x Panipat Local & $36.40 * *$ & -7.63 & 13.33 & $41.67 * *$ & $-9.23 *$ & $13.46^{* *}$ & $-39.60 * *$ & $-29.89 * *$ \\
\hline Punjab-14 x Phule Green & -15.80 & 4.81 & 0.00 & $25.00 * *$ & $-13.85 * *$ & 7.69 & $-52.48 * *$ & $-22.99 * *$ \\
\hline Punjab-14 x Pusa Vishesh & -20.74 & -5.48 & $20.00 * *$ & $50.00 * *$ & 3.45 & $15.38 * *$ & $-20.25 * *$ & $-27.59 * *$ \\
\hline Punjab-14 x Pusa Do Mausami & $\begin{array}{c}- \\
25.77 * *\end{array}$ & 0.41 & $20.00 * *$ & $50.00 * *$ & 0.00 & $11.54 *$ & $-38.89 * *$ & $-24.14 * *$ \\
\hline Punjab-14 x Kalyanpur Barahmasi & 7.20 & 18.59 & 6.67 & $33.33^{* *}$ & $-13.85^{* *}$ & 7.69 & $-26.37 * *$ & $-22.99 * *$ \\
\hline Punjab-14 x Kalyanpur Sona & 4.51 & -0.08 & 0.00 & $25.00 * *$ & -1.72 & $9.62 *$ & -11.84 & $-22.99 * *$ \\
\hline Punjab-14 x Pant Karela-1 & 22.42 & 7.39 & 0.00 & $25.00 * *$ & -3.45 & 7.69 & $-26.74 * *$ & $-27.59 * *$ \\
\hline Arka Harit x Panipat Local & $18.30 *$ & $78.67 * *$ & 0.00 & 16.67 & $-21.54 * *$ & -1.92 & $-27.72 * *$ & $-16.09 *$ \\
\hline Arka Harit x Phule Green & $37.36 * *$ & $107.47 * *$ & 6.67 & $33.33 * *$ & $-18.46^{* *}$ & 1.92 & $-41.84 * *$ & -5.75 \\
\hline Arka Harit x Pusa Vishesh & -10.71 & $34.85 * *$ & 0.00 & 16.67 & -3.64 & 1.92 & -12.66 & $-20.69 * *$ \\
\hline Arka Harit x Pusa Do Mausami & $30.49 * *$ & $97.10 * *$ & 7.14 & $25.00 * *$ & -5.45 & 0.00 & $-22.22 * *$ & -3.45 \\
\hline Arka Harit x Kalyanpur Barahmasi & 13.79 & $71.87 * *$ & -6.67 & 16.67 & $-18.46^{* *}$ & 1.92 & -10.99 & -6.90 \\
\hline Arka Harit x Kalyanpur Sona & 1.43 & $53.20 * *$ & 7.14 & $25.00 * *$ & -1.82 & 3.85 & -10.53 & $-21.84 * *$ \\
\hline Arka Harit x Pant Karela-1 & $24.23 * *$ & $87.63 * *$ & -7.14 & 8.33 & -3.64 & 1.92 & $-17.44 * *$ & $-18.39 * *$ \\
\hline Panipat Local x Phule Green & -2.46 & $41.66^{* *}$ & $-20.00 * *$ & 0.00 & 1.54 & $26.92 * *$ & $-26.95 * *$ & $18.39 * *$ \\
\hline Panipat Local x Pusa Vishesh & $20.57 * *$ & $75.10 * *$ & 15.38 & $25.00 * *$ & 1.54 & $26.92 * *$ & $-18.81 * *$ & -5.75 \\
\hline Panipat Local x Pusa Do Mausami & $19.43^{*}$ & $73.44 * *$ & -7.69 & 0.00 & -1.54 & $23.08 * *$ & -3.70 & $19.54 * *$ \\
\hline Panipat Local x Kalyanpur Barahmasi & $29.71 * *$ & $88.38 * *$ & -6.67 & 16.67 & -4.62 & $19.23 * *$ & 0.00 & $16.09 *$ \\
\hline Panipat Local x Kalyanpur Sona & 2.40 & $48.71 * *$ & -7.69 & 0.00 & 0.00 & $25.00 * *$ & $-17.82 * *$ & -4.60 \\
\hline Panipat Local x Pant Karela-1 & $22.06^{* *}$ & $77.26^{* *}$ & $25.00 * *$ & $25.00 * *$ & -1.54 & $23.08 * *$ & 0.99 & $17.24 * *$ \\
\hline Phule Green x Pusa Vishesh & -6.67 & 16.18 & -13.33 & 8.33 & 0.00 & $25.00 * *$ & $-33.33 * *$ & 8.05 \\
\hline Phule Green x Pusa Do Mausami & 1.96 & $37.93 * *$ & -13.33 & 8.33 & -6.15 & $17.31 * *$ & $-21.28 * *$ & $27.59 * *$ \\
\hline Phule Green x Kalyanpur Barahmasi & 20.80 & $50.37 * *$ & 0.00 & $25.00 * *$ & 0.00 & $25.00 * *$ & $-28.37 * *$ & $16.09 *$ \\
\hline Phule Green x Kalyanpur Sona & 8.87 & $35.52 * *$ & 6.67 & $33.33 * *$ & -4.62 & $19.23 * *$ & $-34.04 * *$ & 6.90 \\
\hline Phule Green x Pant Karela-1 & $43.33 * *$ & $78.42 * *$ & -13.33 & 8.33 & -3.08 & $21.15 * *$ & $-27.66 * *$ & $17.24 * *$ \\
\hline Pusa Vishesh x Pusa Do Mausami & $-23.31 *$ & 3.73 & -15.38 & -8.33 & -1.82 & 3.85 & $-20.37 * *$ & -1.15 \\
\hline Pusa Vishesh x Kalyanpur Barahmasi & -0.70 & 18.42 & $-33.31 * *$ & -16.64 & $-15.38 * *$ & 5.77 & -7.69 & -3.45 \\
\hline Pusa Vishesh x Kalyanpur Sona & -0.56 & 18.59 & $-23.08 * *$ & -16.67 & -7.27 & -1.92 & 2.53 & -6.90 \\
\hline
\end{tabular}




\begin{tabular}{|c|c|c|c|c|c|c|c|c|}
\hline Pusa Vishesh x Pant Karela-1 & 4.38 & 24.48 & 0.00 & 8.33 & -5.45 & 0.00 & -3.49 & -4.60 \\
\hline $\begin{array}{l}\text { Pusa Do Mausami x Kalyanpur } \\
\text { Barahmasi }\end{array}$ & -3.62 & $30.37 *$ & $-20.00 * *$ & 0.00 & $-26.15^{* *}$ & -7.69 & -4.63 & $18.39 * *$ \\
\hline Pusa Do Mausami x Kalyanpur Sona & $26.56 * *$ & $71.20 * *$ & -7.69 & 0.00 & $-15.38 * *$ & $-15.38 * *$ & $-15.74 * *$ & 4.60 \\
\hline Pusa Do Mausami x Pant Karela-1 & 9.94 & $48.71 * *$ & 15.38 & $25.00 * *$ & 0.00 & $-13.46 * *$ & -6.48 & $16.09 *$ \\
\hline $\begin{array}{l}\text { Kalyanpur Barahmasi x Kalyanpur } \\
\text { Sona }\end{array}$ & 1.05 & 11.78 & $20.00 * *$ & $50.00 * *$ & 1.54 & $26.92 * *$ & -2.20 & 2.30 \\
\hline Kalyanpur Barahmasi x Pant Karela-1 & 20.41 & $33.20^{*}$ & 6.67 & $33.33 * *$ & 1.54 & $26.92 * *$ & 3.30 & 8.05 \\
\hline Kalyanpur Sona x Pant Karela-1 & -2.34 & -6.64 & $38.46 * *$ & $50.00 * *$ & 1.92 & 1.92 & -2.33 & -3.45 \\
\hline
\end{tabular}

Kalyanpur Sona x Pant Karela-

BP Better Parent

CC Commerical Check

\begin{tabular}{|c|c|c|c|c|c|c|c|c|}
\hline \multirow[t]{2}{*}{ Hybrids } & \multicolumn{2}{|c|}{ Fruit weight (g) } & \multicolumn{2}{|c|}{$\begin{array}{l}\text { Fruit diameter } \\
(\mathrm{cm})\end{array}$} & \multicolumn{2}{|c|}{$\begin{array}{c}\text { Total fruit yield per } \\
\text { vine(kg) }\end{array}$} & \multicolumn{2}{|c|}{ Ascorbic acid (mg/100g) } \\
\hline & BP & $\mathrm{CC}$ & $\mathrm{BP}$ & $\mathrm{CC}$ & BP & $\mathrm{CC}$ & BP & $\mathrm{CC}$ \\
\hline Punjab-14 x Arka Harit & -3.57 & $-10.00 *$ & -9.09 & -13.04 & $9.68 * *$ & $21.43 * *$ & 2.56 & -2.44 \\
\hline Punjab-14 x Panipat Local & $-9.68 *$ & -6.67 & $-15.38 *$ & -4.35 & $-6.45 *$ & 3.57 & -2.50 & -4.88 \\
\hline Punjab-14 x Phule Green & $-27.27 * *$ & $-20.00 * *$ & $-50.00 * *$ & $-43.48 * *$ & $-6.45^{*}$ & 3.57 & 5.13 & 0.00 \\
\hline Punjab-14 x Pusa Vishesh & -3.70 & $-13.33 * *$ & -9.52 & $-17.39 *$ & $-9.68 * *$ & 0.00 & 2.56 & -2.44 \\
\hline Punjab-14 x Pusa Do Mausami & $-12.90 * *$ & $-10.00 *$ & $-33.33 * *$ & $-39.13 * *$ & -3.23 & $7.14 *$ & 5.13 & 0.00 \\
\hline Punjab-14 x Kalyanpur Barahmasi & -3.70 & $-13.33 * *$ & $-33.33 * *$ & $-39.13 * *$ & 0.00 & $10.71 * *$ & 0.00 & -4.88 \\
\hline Punjab-14 x Kalyanpur Sona & 3.85 & $-10.00 *$ & $-23.81 * *$ & $-30.43 * *$ & $-6.45^{*}$ & 3.57 & 0.00 & -4.88 \\
\hline Punjab-14 x Pant Karela-1 & $-11.11 *$ & $-20.00 * *$ & $-18.18 *$ & $-21.74 * *$ & $-9.68 * *$ & 0.00 & 2.56 & -2.44 \\
\hline Arka Harit x Panipat Local & -6.45 & -3.33 & -7.69 & 4.35 & 3.23 & $14.29 * *$ & -5.00 & -7.32 \\
\hline Arka Harit x Phule Green & $-12.12 * *$ & -3.33 & $-26.92 * *$ & $-17.39 *$ & $-9.68 * *$ & 0.00 & 2.78 & -9.76 \\
\hline Arka Harit x Pusa Vishesh & -3.57 & $-10.00 *$ & -4.55 & -8.70 & $-6.45^{*}$ & 3.57 & 2.78 & -9.76 \\
\hline Arka Harit x Pusa Do Mausami & -3.23 & 0.00 & -9.09 & -13.04 & $-12.90 * *$ & -3.57 & 5.56 & -7.32 \\
\hline Arka Harit x Kalyanpur Barahmasi & $-10.71 *$ & $-16.67 * *$ & -13.64 & $-17.39 *$ & $-16.13 * *$ & $-7.14 *$ & 0.00 & -12.20 \\
\hline Arka Harit x Kalyanpur Sona & $-14.29 * *$ & $-20.00 * *$ & -13.64 & $-17.39 *$ & $-16.13 * *$ & $-7.14 *$ & 2.78 & -9.76 \\
\hline Arka Harit x Pant Karela-1 & -7.14 & $-13.33 * *$ & -9.09 & -13.04 & $-12.90 * *$ & -3.57 & 5.56 & -7.32 \\
\hline
\end{tabular}




\begin{tabular}{|c|c|c|c|c|c|c|c|c|}
\hline Panipat Local x Phule Green & $-9.09 *$ & 0.00 & $-19.23 * *$ & -8.70 & $-9.68 * *$ & 0.00 & 0.00 & -2.44 \\
\hline Panipat Local x Pusa Vishesh & $-9.68 *$ & -6.67 & $-23.08 * *$ & -13.04 & $-16.13 * *$ & $-7.14^{*}$ & -2.50 & -4.88 \\
\hline Panipat Local x Pusa Do Mausami & 0.00 & 3.33 & -11.54 & 0.00 & $-6.45^{*}$ & 3.57 & 2.50 & 0.00 \\
\hline Panipat Local x Kalyanpur Barahmasi & -6.45 & -3.33 & $-19.23 * *$ & -8.70 & $-12.90 * *$ & -3.57 & 0.00 & -2.44 \\
\hline Panipat Local x Kalyanpur Sona & $-12.90 * *$ & $-10.00 *$ & $-23.08 * *$ & -13.04 & $-12.90 * *$ & -3.57 & 0.00 & -2.44 \\
\hline Panipat Local x Pant Karela-1 & -6.45 & -3.33 & $-19.23 * *$ & -8.70 & $-6.45^{*}$ & 3.57 & 2.50 & 0.00 \\
\hline Phule Green x Pusa Vishesh & $-12.12 * *$ & -3.33 & $-19.23 * *$ & -8.70 & 0.00 & 3.57 & 2.86 & -12.20 \\
\hline Phule Green x Pusa Do Mausami & -6.06 & 3.33 & $-15.38^{*}$ & -4.35 & 3.45 & $7.14^{*}$ & 0.00 & $-14.63 *$ \\
\hline Phule Green x Kalyanpur Barahmasi & $-15.15 * *$ & -6.67 & $-26.92 * *$ & $-17.39 *$ & $-6.90 *$ & -3.57 & 5.71 & -9.76 \\
\hline Phule Green x Kalyanpur Sona & $-15.15^{* *}$ & -6.67 & $-23.08 * *$ & -13.04 & $-6.90 *$ & -3.57 & 0.00 & $-14.63 *$ \\
\hline Phule Green x Pant Karela-1 & $-9.09 *$ & 0.00 & $-26.92 * *$ & $-17.39 *$ & -3.45 & 0.00 & 2.86 & -12.20 \\
\hline Pusa Vishesh x Pusa Do Mausami & -3.23 & 0.00 & -9.52 & $-17.39 *$ & -3.57 & -3.57 & 8.57 & -7.32 \\
\hline Pusa Vishesh x Kalyanpur Barahmasi & 0.00 & $-10.00 * *$ & -9.92 & $-21.67 * *$ & 0.00 & $-7.14 *$ & 0.00 & $-14.63^{*}$ \\
\hline Pusa Vishesh x Kalyanpur Sona & -3.70 & $-13.33 * *$ & -10.53 & $-26.09 * *$ & $-7.69 *$ & $-14.29 * *$ & 2.86 & -12.20 \\
\hline Pusa Vishesh x Pant Karela-1 & 0.00 & $-10.00 *$ & -13.64 & $-17.39 *$ & $-13.79 * *$ & $-10.71 * *$ & 0.00 & $-14.63^{*}$ \\
\hline $\begin{array}{l}\text { Pusa Do Mausami x Kalyanpur } \\
\text { Barahmasi }\end{array}$ & -6.45 & -3.33 & 0.00 & -8.70 & 0.00 & 0.00 & 0.00 & $-21.95 * *$ \\
\hline Pusa Do Mausami x Kalyanpur Sona & -3.23 & 0.00 & -9.52 & $-17.39 *$ & -3.57 & -3.57 & 3.13 & $-19.51 * *$ \\
\hline Pusa Do Mausami x Pant Karela-1 & -6.45 & -3.33 & -9.09 & -13.04 & -3.45 & 0.00 & -3.12 & $-24.39 * *$ \\
\hline $\begin{array}{l}\text { Kalyanpur Barahmasi x Kalyanpur } \\
\text { Sona }\end{array}$ & 0.00 & $-10.00 *$ & 15.00 & 0.00 & 3.85 & -3.57 & 3.33 & $-24.39 * *$ \\
\hline Kalyanpur Barahmasi x Pant Karela-1 & 3.70 & -6.67 & -4.55 & -8.70 & $-10.34 * *$ & $-7.14 *$ & -3.33 & $-29.27 * *$ \\
\hline Kalyanpur Sona x Pant Karela-1 & 3.70 & -6.67 & -4.55 & -8.70 & $-6.90 *$ & -3.57 & 10.00 & $-19.51 * *$ \\
\hline
\end{tabular}

*Singnificant at 5\% * Singnificant at 1\% BP Better Parent

CC Commerical Check 
For days to fruit harvest 9 and 2 cross over better parent and standard variety showed negative heterosis, Pusa Do Mousami $\mathrm{x}$ kalyanpur Baramasi over better parent and Pusa Do Mousami x kalyanpur Sona showed standard heterosis for days to fruit harvest. For fruit length heterosis 9 cross over standard variety showed positive significant heterosis. Panipat Local x Phule Green and kalyanpur Baramasi showed high degree of standard heterosis.

Chaubey and Ram (2004) For fruit weight none of cross over better parent and standard variety showed positive and significant heterosis likewise for fruit diameter none of cross over better parent and standard variety showed positive and significant heterosis by Talekar et al., (2013) and Naliyadhara et al., (2010). For total fruit yield per vine 1 and 5 cross over better parent and standard variety showed positive and significant heterosis.

The high degree of standard heterosis was observed in cross Punjab-14 x Arka Harit by Thangamani et al., (2011).For Ascorbic content heterosis none of cross over better parent and standard variety showed heterosis in any direction while highest standard heterosis was observed in cross kalyanpur Baramasi x Pant Karela-1.

\section{References}

Chaubey, A. K. and Ram, H.H. 2004. Heterosis for fruit yield and its components in bitter gourd (Momordica charantia. L.). Vegetable Science 31(1):51-53.

Fonseca, S. and Patterson, P.L. 1968. Hybrid vigour in seven parent diallel cross in common winter wheat (Triticum aestivum L.).Crop Science. 8:85-88.

Khattra, A. S, Singh, N. J. and Thakar, J. C. 2000. Studies on combining ability in bitter gourd. Vegetable Science.21:158-162

Kumara, B. S., Puttarau, T. B., Hongal, S., Prakash, K.J. and Sudheesh, N.K. 2011. Combining ability studies in bitter gourd (Momordica charantia L.) for quantitative characters. The Asian Journal of Horticulture; 6No.1:135140.

Mangal, J. L., Dixit, J., Pandita, M. L. and Sindhu, A. S. 1984. Genetic variability and correlation studies in bitter gourd (Momordica charantia L.). Indian Journal of Horticulture. 39: 94-99.

Naliyadhara, M.V, Dhaduk, L.K, Aubard,K. and Mehta, D.R. 2010. Combinig ability analysis in sponge gourd [Luffa cylindrical (Roem) L.] Vegetable Science 37(1):21-24.

Ram, D., Kalloo,G. and Singh, M. 1999. Combining ability of quantitive characters in bitter gourd (Momordica charantia L.) Indian Journal of Agriculture Science 69:122-127.

Singh, A. K., Pan, R. S. and Bhavana, P. 2013. Heterosis and combining ability analysis in bitter gourd (Momordica charantia L.) AnInternational Quarterly Journal of Life Science 8(4):1533-1536.

Thangamani, C., Pugalendhi. L., Sumathi. T. and Rsvitha, 2011. Estimation of combining ability and heterosis for yield and quality characters in bitter gourd

Talekar. N. S., Vaddoria, M. A. and Kalkaran, G. V. 2013. Heterosis studies for quantitative traits in bitter gourd (Momordica charantia L.)Progressive Research 8: 650-653.

Verma, R. S. and Singh, M. K. 2014. Studies on heterosis for yield and its component of bitter gourd (Mommordica charantia L.). The Asian Journal of Horticulture 9:217223.

\section{How to cite this article:}

Bhatt, L., S.P. Singh, A.K. Soni and Samota, M.K. 2017. Studies on Heterosis in Bitter Gourd (Momordica charantia L.). Int.J.Curr.Microbiol.App.Sci. 6(7): 4069-4077.

doi: https://doi.org/10.20546/ijcmas.2017.607.422 